\title{
Correction to: Network Effects of International Shocks and Spillovers
}

\author{
Alexei Kireyev $^{1}$ - Andrei Leonidov ${ }^{2}$ \\ Published online: 26 June 2018 \\ (C) Springer Science+Business Media, LLC, part of Springer Nature 2018
}

\section{Correction to: Netw Spat Econ. https://doi.org/10.1007/s11067-018-9400-7}

The original version of the article unfortunately contained a mistake. The Annex table was distorted and some columns were out of order. The corrected version is presented below.

The online version of the original article can be found at https://doi.org/10.1007/s11067-018-9400-7

\section{Alexei Kireyev \\ akireyev@hotmail.com}

1 International Monetary Fund, Washington, DC, USA

2 Lebedev Physics Institute, Moscow, Russia 


\begin{tabular}{|c|c|c|c|c|c|c|c|c|c|c|c|c|c|c|}
\hline & Ann & ex 1. & Sho & ock & Pass-Through & Coeffic & cient: & $s: F$ & ull C & Country Class & sificati & tion & & \\
\hline & $\begin{array}{l}\text { Pass- } \\
\text { through }\end{array}$ & GDP per & Trade b & $\begin{array}{r}\text { Doing } \\
\text { business }\end{array}$ & & $\begin{array}{r}\begin{array}{r}\text { Pass- } \\
\text { through }\end{array} \\
\end{array}$ & GDP per & Trade & $\begin{array}{r}\text { Doing } \\
\text { business }\end{array}$ & & $\begin{array}{c}\begin{array}{c}\text { Pass- } \\
\text { through }\end{array} \\
\text { the }\end{array}$ & GDP per & & $\begin{array}{r}\text { Doing } \\
\text { business }\end{array}$ \\
\hline Spillover amplifiers & & & & rating & Spillover absorbers & & & openness & rating & Spillover blockers & coefficient & capita o & openness & rating \\
\hline Argentina & 1.68 & 14,789 & 29 & 124 & Moldova & 0.99 & 4,521 & 125 & 63 & Algeria & 0.00 & 12,893 & 63 & 154 \\
\hline Thailand & 1.46 & 13,932 & 144 & 26 & || Slovenia & 0.99 & 27,368 & 143 & 51 & Angola & 0.00 & 7,488 & 96 & 181 \\
\hline Korea & 1.32 & 32,708 & 103 & & |Japan & 0.96 & 35,614 & 35 & 29 & Azerbaijan & 0.00 & 16,593 & 76 & 80 \\
\hline Hong Kong SAR & 1.29 & 51,509 & 458 & 3 & I celand & 0.95 & 40,789 & 103 & 12 & Belarus & 0.00 & 17,055 & 125 & 57 \\
\hline Denmark & 1.29 & 42,483 & 103 & 4 & Germany & 0.95 & 42,884 & 85 & 14 & Belgium & 0.00 & 40,609 & 164 & 42 \\
\hline Indonesia & 1.29 & 9,254 & 49 & 114 & $\begin{array}{l}\text { France } \\
\text { Frant }\end{array}$ & 0.92 & 37,217 & 58 & 31 & Belize & 0.00 & 8,215 & 127 & 118 \\
\hline India & 1.27 & 5,244 & 53 & $\begin{array}{l}142 \\
142\end{array}$ & $\mid \begin{array}{l}\text { Ireland } \\
\text { irend }\end{array}$ & 0.92 & 44,647 & $\begin{array}{r}190 \\
190\end{array}$ & $\begin{array}{l}13 \\
13\end{array}$ & Benin & 0.00 & $\begin{array}{l}0 \\
1,733\end{array}$ & 52 & $\begin{array}{l}151 \\
151\end{array}$ \\
\hline Ukraine & 1.22 & 8,508 & 102 & $\begin{array}{r}96 \\
96\end{array}$ & Colombia & 0.92 & 12,025 & 38 & 34 & Bosnia and Herzegovina & 0.00 & 9,387 & 85 & 107 \\
\hline Sri Lanka & 1.20 & 9,426 & 54 & 99 & Czech Republic & 0.91 & 28,124 & 149 & 44 & Burkina Faso & 0.00 & 1,630 & 57 & 167 \\
\hline Turkey & 1.18 & 18,567 & 58 & 55 & Peru & 0.90 & 11,396 & 48 & 35 & Burundi & 0.00 & 747 & 42 & 152 \\
\hline Romania & 1.17 & 18,184 & 85 & 48 & sweden & 0.89 & 43,540 & 83 & 11 & Cambodia & 0.00 & 2,944 & 140 & 135 \\
\hline Malaysia & 1.17 & 22,589 & 154 & 18 & Singapore & 0.89 & 76,237 & 358 & $\begin{array}{l}1 \\
1\end{array}$ & Cape Verde & 0.00 & 6,210 & 87 & 122 \\
\hline Croatia & 1.11 & 20,049 & 85 & 65 & Austria & 0.87 & 44,056 & 103 & 21 & Central African Republic & 0.00 & 584 & 33 & 187 \\
\hline Estonia & 1.11 & 25,254 & 171 & 17 & || Portugal & 0.82 & 25,933 & 78 & 25 & Chad & 0.00 & 2,022 & 70 & 185 \\
\hline Latvia & 1.08 & 21,833 & 122 & 23 & Barbados & 0.81 & 14,917 & 97 & 106 & Comoros & 0.00 & 1,400 & 78 & 159 \\
\hline Switzerland & 1.08 & 54,993 & 132 & 20 & ||china & 0.79 & 11,525 & 50 & 90 & Costa Rica & 0.00 & 13,431 & 74 & 83 \\
\hline Poland & 1.07 & 22,835 & 90 & 32 & || Mongolia & 0.77 & 9,132 & 112 & 72 & Côte d'Ivoire & 0.00 & 3,107 & 91 & 147 \\
\hline |israel & 1.07 & 30,927 & 64 & 40 & Bulgaria & 0.75 & 15,695 & 137 & 38 & Cyprus & 0.00 & 27,394 & 78 & 64 \\
\hline |'taly & 1.07 & 33,924 & 55 & 56 & Russia & 0.74 & 23,564 & 51 & 62 & Dem. Rep. of Congo & 0.00 & & & 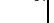 \\
\hline Finland & 1.06 & 38,821 & 77 & 9 & Uzbekistan & 0.73 & 5,002 & 59 & 141 & Djibouti & 0.00 & 2,903 & & 155 \\
\hline United States & 1.05 & 51,340 & 30 & 7 & Hungary & 0.73 & 22,707 & 170 & 54 & Dominica & 0.00 & 10,011 & 81 & 97 \\
\hline spain & 1.05 & 31,683 & 60 & 33 & | Guyana & 0.72 & 6,336 & 65 & 123 & Dominican Republic & 0.00 & 11,795 & 57 & 84 \\
\hline Lithuania & 1.05 & 24,470 & 156 & 24 & Jamaica & 0.72 & 8,607 & 83 & 58 & Ecuador & 0.00 & 10,541 & 61 & 115 \\
\hline Pakistan & 1.05 & 4,454 & 33 & 128 & Vietnam & 0.72 & 5,125 & 164 & 78 & Egypt & 0.00 & 10,733 & 42 & 112 \\
\hline Brazil & 1.04 & 14,555 & 28 & 120 & Philippines & 0.71 & 6,326 & 60 & 95 & Equatorial Guinea & 0.00 & 32,685 & 157 & 165 \\
\hline Uruguay & 1.03 & 18,966 & 51 & 82 & Greece & 0.71 & 24,305 & 63 & 61 & Ethiopia & 0.00 & 1,336 & 42 & 132 \\
\hline Slovak Republic & 1.03 & 25,759 & 181 & 37 & $\begin{array}{l}\text { chile } \\
\text { Chile }\end{array}$ & 0.71 & 21,714 & 65 & ${ }_{41}$ & Fiji & 0.00 & 7,502 & 136 & 81 \\
\hline New Zealand & 1.03 & 33,020 & 57 & 2 & || Mexico & 0.70 & 16,291 & 64 & 39 & Georgia & 0.00 & 6,930 & 102 & 15 \\
\hline Kyrgyz Republic & 1.01 & 3,110 & 143 & 102 & South Africa & 0.69 & 12,454 & 64 & 43 & Grenada & 0.00 & 11,272 & 76 & 126 \\
\hline Average & 1.16 & 24,248 & 101 & 53 & | Canada & 0.69 & 41,899 & 62 & 16 & Guinea & 0.00 & 1,213 & 83 & 169 \\
\hline & & & & & | Armenia & 0.68 & 7,527 & 75 & ${ }_{45}$ & Guinea-Bissau & 0.00 & 1,362 & & 179 \\
\hline & & & & & Netherlands & 0.68 & 45,021 & 156 & 27 & Hatio & 0.00 & 1,648 & 71 & 180 \\
\hline & & & & & United Kingdom & 0.67 & 36,931 & 62 & 8 & Honduras & 0.00 & 4,445 & 117 & 104 \\
\hline & & & & & Albania & 0.67 & 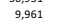 & 88 & 68 & Islamic Republic of Afghanistan & 0.00 & 1,884 & 55 & 183 \\
\hline & & & & & Senegal & 0.66 & 2,170 & 74 & 161 & Islamic Republic of Iran & 0.00 & $\begin{array}{r}1,004 \\
15,090\end{array}$ & & $\begin{array}{l}130 \\
130\end{array}$ \\
\hline & & & & & $\begin{array}{l}\text { Sentrgi } \\
\text { Australia }\end{array}$ & 0.66 & $\begin{array}{l}2,170 \\
42,834\end{array}$ & 41 & $\begin{array}{r}101 \\
10\end{array}$ & 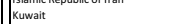 & 0.00 & $\begin{array}{l}1,3,190 \\
53,197\end{array}$ & 98 & $\begin{array}{r}830 \\
86\end{array}$ \\
\hline & & & & & $\begin{array}{l}\text { Tujujistan } \\
\text { Tajtan }\end{array}$ & 0.65 & 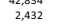 & 88 & 166 & Lao People's Democratic Republic & 0.00 & $\begin{array}{l}33,197 \\
4,667\end{array}$ & 83 & $\begin{array}{r}80 \\
148\end{array}$ \\
\hline & & & & & Former Yugoslav Republic of Macedonia & 0.64 & 11,609 & 127 & 30 & Lebanon & 0.00 & 16,623 & 139 & 104 \\
\hline & & & & & Bangladesh & 0.62 & 2,853 & 46 & 173 & Liberia & 0.00 & 850 & 122 & 174 \\
\hline & & & & & Solomon Islands & 0.60 & 2,003 & 119 & $\begin{array}{r}87 \\
87\end{array}$ & Luxembourg & 0.00 & 88,850 & 371 & 59 \\
\hline & & & & & Papua New Guinea & 0.60 & 2,558 & & 133 & Malawi & 0.00 & 755 & 111 & 164 \\
\hline & & & & & Kazakhstan & 0.60 & 22,470 & 65 & 77 & Maldives & 0.00 & 11,283 & 222 & 116 \\
\hline & & & & & Malta & 0.58 & 28,822 & 183 & 94 & Mali & 0.00 & $\begin{array}{r}1,589 \\
1,50\end{array}$ & 69 & 146 \\
\hline & & & & & Tunisia & 0.57 & 10,768 & 103 & 60 & Mauritius & 0.00 & 17,146 & 121 & 28 \\
\hline & & & & & Libya & 0.53 & 20,371 & & 188 & Mozambique & 0.00 & 1,070 & 71 & 127 \\
\hline & & & & & Bolvia & 0.52 & 5,934 & 81 & 157 & Myanmar & 0.00 & & & 177 \\
\hline & & & & & Brunei Darussalam & 0.50 & 69,474 & 109 & 101 & Nepal & 0.00 & 2,173 & 48 & 108 \\
\hline & & & & & $\begin{array}{l}\text { Jordan } \\
\text { Jostatis }\end{array}$ & 0.49 & 11,405 & 114 & 117 & Nicaragua & 0.00 & 4,494 & 93 & 119 \\
\hline & & & & & Kenya & 0.48 & 2,705 & 51 & 136 & Niger & 0.00 & 887 & 65 & 168 \\
\hline & & & & & Ghana & 0.46 & 3,864 & 89 & $\begin{array}{r}70 \\
70\end{array}$ & Nigeria & 0.00 & 5,423 & 31 & 170 \\
\hline & & & & & Trinidad and Tobago & $\begin{array}{l}0.46 \\
0.46\end{array}$ & $\begin{array}{r}3,864 \\
29,469\end{array}$ & 103 & $\begin{array}{l}7 \\
79\end{array}$ & $\mid \begin{array}{l}\text { Pvenama } \\
\text { Panama }\end{array}$ & 0.00 & $\begin{array}{r}3,423 \\
18,793\end{array}$ & $\begin{array}{r}31 \\
155\end{array}$ & 52 \\
\hline & & & & & Venezuela & 0.45 & 17,615 & & 182 & Paraguay & 0.00 & 7,833 & 94 & ${ }_{92}$ \\
\hline & & & & & United Arab Emirates & $\begin{array}{l}0.45 \\
0.44\end{array}$ & 17,615 & 176 & 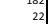 & 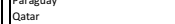 & 0.00 & $\begin{array}{r}1,833 \\
127,562\end{array}$ & $\begin{array}{l}94 \\
97\end{array}$ & $\begin{array}{l}92 \\
50\end{array}$ \\
\hline & & & & & Gabon & 0.42 & 18,646 & 94 & 144 & Republic of Congo & 0.00 & 5,680 & 143 & 178 \\
\hline & & & & & Norway & 0.41 & 62,411 & 67 & 6 & Republic of Yemen & 0.00 & 3,832 & & 137 \\
\hline & & & & & Cameroon & 0.37 & 2,739 & 50 & 158 & Rwanda & 0.00 & 1,426 & 45 & 46 \\
\hline & & & & & Oman & 0.37 & 21,913 & 99 & 66 & Samoa & 0.00 & 5,584 & 81 & 67. \\
\hline & & & & & Zambia & 0.35 & 3,800 & 82 & 111 & são Tomé and Príncipe & 0.00 & 2,876 & 58 & 153 \\
\hline & & & & & Morocco & 0.35 & 6,967 & 81 & 71 & Seychelles & 0.00 & 23,799 & 164 & 85 \\
\hline & & & & & & 0.35 & 14,471 & 67 & 156 & Sierra Leone & 0.00 & 1,495 & 108 & 140 \\
\hline & & & & & Madagascar & 0.33 & 1,369 & 73 & 163 & St. Kitts and Nevis & 0.00 & 20,709 & 88 & 121 \\
\hline & & & & & Mauritania & 0.33 & 2,945 & 134 & 176 & St. Lucia & 0.00 & 10,152 & 97 & 100 \\
\hline & & & & & Bahrain & 0.32 & $\begin{array}{r}2,4434 \\
42,444\end{array}$ & 122 & $\begin{array}{r}5 \\
53\end{array}$ & St. Vincent and the Grenadines & 0.00 & 10,154 & 87 & 103 \\
\hline & & & & & The Bahamas & 0.32 & 22,518 & 98 & 97 & Sudan & 0.00 & $\begin{array}{l}3,265 \\
3,265\end{array}$ & 26 & 160 \\
\hline & & & & & $\begin{array}{l}\text { The bandarias } \\
\text { Uganda }\end{array}$ & 0.31 & $\begin{array}{r}1,621 \\
1,621\end{array}$ & 51 & $\begin{array}{r}91 \\
150\end{array}$ & Tanzania & 0.00 & $\begin{array}{l}3,203 \\
2,365\end{array}$ & $\begin{array}{l}26 \\
49\end{array}$ & ${ }_{131}$ \\
\hline & & & & & Guatemala & 0.25 & 7,063 & 59 & 73 & The Gambia & 0.00 & 1,608 & 88 & 138 \\
\hline & & & & & El Salvador & 0.25 & 7,515 & 72 & 109 & Togo & 0.00 & 1,346 & 96 & 149 \\
\hline & & & & & Syrian Arab Republic & 0.23 & & & 175 & Tonga & 0.00 & 5,134 & 80 & 69 \\
\hline & & & & & Saudi Arabia & 0.22 & 52,068 & 82 & 49 & Turkmenistan & 0.00 & 13,555 & 118 & \\
\hline & & & & & Suriname & 0.14 & 15,556 & & 162 & Vanuatu & 0.00 & 2,895 & 99 & 76 \\
\hline & & & & & Average & 0.62 & 20,482 & 94 & 80 & Zimbabwe & 0.00 & $\begin{array}{r}1,773 \\
11386\end{array}$ & 86 & 171 \\
\hline
\end{tabular}

The original article has been corrected. 